\title{
Laboratory Response to Ebola — West Africa and United States
}

\author{
Tara K. Sealy, MSc ${ }^{1}$ \\ Bobbie R. Erickson, $\mathrm{MPH}^{1}$ \\ Céline H. Taboy, $\mathrm{PhD}^{1}$ \\ Ute Ströher, $\mathrm{PhD}^{1}$ \\ Jonathan $\mathrm{S}$. Towner, $\mathrm{PhD}^{1}$ \\ Sharon E. Andrews, $\mathrm{MPH}^{1}$ \\ Laura E. Rose, MTS $^{2}$ \\ Elizabeth Weirich ${ }^{3}$ \\ Luis Lowe, $\mathrm{PhD}^{2}$ \\ John D. Klena, $\mathrm{PhD}^{1}$ \\ Christina F. Spiropoulou, $\mathrm{PhD}^{1}$ \\ Mark A. Rayfield, $\mathrm{PhD}^{4}$ \\ Brian H. Bird, DVM, PhD ${ }^{1}$

\begin{abstract}
${ }^{1}$ Division of High-Consequence Pathogens and Pathology, National Center for Emerging and Zoonotic Infectious Diseases, CDC ${ }^{2}$ Division of Preparedness and Emerging Infections, National Center for Emerging and Zoonotic Infectious Diseases, CDC ${ }^{3}$ Division of Laboratory Services, Center for Surveillance, Epidemiology and Laboratory Services, CDC

${ }^{4}$ Division of Global Health Protection, Center for Global Health, CDC
\end{abstract}

Corresponding author: Mark A. Rayfield, Division of Global Health Protection, Center for Global Health, CDC. Telephone: 404-718-4506; E-mail: mrayfield@cdc.gov.

\section{Summary}

The 2014-2016 Ebola virus disease (Ebola) epidemic in West Africa highlighted the need to maintain organized laboratory systems or networks that can be effectively reorganized to implement new diagnostic strategies and laboratory services in response to large-scale events. Although previous Ebola outbreaks enabled establishment of critical laboratory practice safeguards and diagnostic procedures, this Ebola outbreak in West Africa highlighted the need for planning and preparedness activities that are better adapted to emerging pathogens or to pathogens that have attracted little commercial interest. The crisis underscored the need for better mechanisms to streamline development and evaluation of new diagnostic assays, transfer of material and specimens between countries and organizations, and improved processes for rapidly deploying health workers with specific laboratory expertise. The challenges and events of the outbreak forced laboratorians to examine not only the comprehensive capacities of existing national laboratory systems to recognize and respond to events, but also their sustainability over time and the mechanisms that need to be pre-established to ensure effective response. Critical to this assessment was the recognition of how response activities (i.e., infrastructure support, logistics, and workforce supplementation) can be used or repurposed to support the strengthening of national laboratory systems during the postevent transition to capacity building and recovery. This report compares CDC's domestic and international laboratory response engagements and lessons learned that can improve future responses in support of the International Health Regulations and Global Health Security Agenda initiatives.

The activities summarized in this report would not have been possible without collaboration with many U.S. and international partners (http://www.cdc.gov/vhflebolaloutbreaks/2014-west-africalpartners.html).

\section{Introduction}

The 2014-2016 Ebola virus disease (Ebola) epidemic in West Africa presented an unprecedented challenge for CDC and its partners, not only because of the complexity of responding to an Ebola outbreak of such proportion, duration, and intensity internationally, but also because of the exceptional situation created for U.S. clinical and public health laboratory systems. CDC's role in health emergencies is complex; multilayered interactions range from state and local health care institutions and care providers to the national-level U.S. agencies and ministries of health internationally.
As with many other viral hemorrhagic fevers, Ebola can be difficult to differentiate clinically from other common infectious diseases and requires laboratory diagnostics to confirm or rule out Ebola virus (EBOV) infection when the level of suspicion is heightened. Rapid and reliable laboratory testing for diagnosis of suspected Ebola cases or of EBOV-infected persons is central to controlling the disease and serves multiple purposes, including differential diagnosis of cases and triage of patients into care, initiation of contact tracing, and safe discharge of EBOV-negative patients to their home communities. 
The increase in cases during March-October 2014 led to an influx of international support to the three most affected countries (Guinea, Liberia, and Sierra Leone) as well as to Mali and Nigeria. The World Health Organization initially organized laboratory response activities as part of the Emerging and Dangerous Pathogens Laboratory Network and Global Outbreak Alert and Response Network (1).

These early activities were hampered by several factors. These included the lack of approved diagnostic tools adapted to such a large-scale outbreak; a shortage of skilled personnel; limited biosafety knowledge by local staff; inadequate supply chain management to provide for universal use of personal protective equipment; and weak national laboratory systems that could not support the rollout of standardized methods and mechanisms for safe collection, transport, and testing of specimens from persons suspected to have Ebola.

\section{Laboratory Environment}

\section{International Response}

Historically, the remote locations of Ebola outbreaks have required rapid, highly mobile, and transient responses. This changed with the recent Ebola epidemic in West Africa. From the early serology studies that retrospectively mapped the extent of the 1995 Ebola outbreak in Kikwit (2), recognition of the need to establish field laboratory capacities grew. By 2000, CDC's Viral Special Pathogens Branch field laboratory in Gulu, Uganda, was able to provide next-day serology and reverse transcription-polymerase chain reaction (RT-PCR) diagnostics on specimens for acute and convalescent case identification (3). This represented a transition for field laboratories from retrospectively mapping the extent of an outbreak to providing near real-time diagnostic service. These field techniques continued to be polished during later responses and ecologic studies that sought an animal reservoir for the virus. By 2005, the concept of a high-throughput field laboratory using semiautomated systems was firmly established $(4,5)$. These field laboratories had become highly sophisticated but still could be broken down into a series of trunks that could be transported readily by one or two persons and set up in local structures (e.g., health care unit, house, or tent).

The greatest challenge to robust field operations was not the selection and arrangement of diagnostic equipment but rather the ability to ensure biosecurity and safety for staff during operations. These concerns were satisfied by physically separating sample processing of infectious materials from the remainder of the testing procedure and having the staff operate in complete personal protective equipment, including a powered air-purifying respirator or an N95 respirator to guard against unexpected exposures to infectious materials (G). This was the standard of operations in the earliest international laboratories responding to the Ebola outbreak in summer 2014, and many laboratories continued to operate in this manner. The CDC laboratory originally established under the Global Outbreak Alert and Response Network in Kenema District and later moved to Bo District, Sierra Leone, is one such example. Located in a small house, this laboratory provided rapid results for approximately 26,000 specimens, with a peak of 180 specimens in a single day. Diagnostic operations were maintained continuously from August 2014 until the facility closed in late October 2015, even during the 70-km transition from Kenema to Bo (7). These earlier lessons would help countries affected by Ebola develop recovery and capacity plans for both sustainable fixed laboratory structures and rapid response strategies in their national laboratory systems.

\section{Domestic Response}

During the domestic response to Ebola, the U.S. clinical laboratory environment presented a different set of challenges from those in West Africa. In the United States, many years of preparation for such an event, including coordinated preparedness activities, produced robust and adaptable laboratory systems capable of rapidly deploying new assays and technologies. This preparedness enabled ready adaptation of existing clinical and public health laboratory networks to respond to the need to scale up diagnostic testing for Ebola. However, many clinical laboratories had migrated to largescale high-volume laboratory structures that rely heavily on rapid specimen transport and reporting systems, often across multiple states. Regional facilities and laboratories in larger hospitals have open working spaces, high-throughput automated systems, robotic equipment, and multiple parallel testing of samples. This environment is not readily conducive to the introduction of isolated specimen-specific management and safeguard measures for samples from persons with suspected EBOV infection, and the multiple-barrier protective practices employed in field laboratories are not readily adaptable to these large open-floor-plan environments. These considerations increased concerns about and difficulties in standardizing biocontainment and safety procedures for routine clinical testing in open laboratory environments. Manufacturers could not guarantee the decontamination procedures for their products and announced they would void warranties on products used in Ebola care and treatment. The costs and liability concerns led many large referral laboratories to announce they would not accept routine clinical test specimens from persons suspected to have Ebola. These concerns within the health care facilities and referral laboratories initially led 
to delays in routine diagnostic services to patients in whom Ebola was a concern $(8,9)$.

CDC and public health partners worked closely with the public and private clinical laboratory sectors to establish guidance for managing and testing routine clinical specimens in situations where concern existed about EBOV infection (10). In addition, a tiered service model was established for clinical institutions and their laboratories that assess and provide care for patients exhibiting symptoms of possible Ebola or who were known to be infected with EBOV (11). State public health agencies identified and designated these geographically distributed facilities. Adjustments to the laboratory environment resulted from the collaborative engagement of multiple individuals and organizations, through peer-reviewed reports, national conference calls, webinars, electronic messaging and listserves, consults through professional associations, and other communications media. This collaboration was one of the greatest strengths of the response, particularly as it pertains to the laboratory.

CDC collaborated with the U.S. Department of Defense (DoD), the Association of Public Health Laboratories, and state and local public health agencies to meet the need for enhanced domestic diagnostic capacity by rapidly expanding EBOV testing within the Laboratory Response Network (LRN) (12). This network enabled quick distribution of testing capacity to well-equipped laboratories serving the entire United States, with staff trained to manage dangerous pathogens and operate under uniform practices, and established processes of communications with public health institutions.

The initial deployment of the DoD Ebola Zaire quantitative real-time polymerase chain reaction (EZ1 rRT-PCR) began in early August 2014 with 13 LRN laboratories. CDC selected these laboratories based on the known population of West African citizens in the area and their proximity to major airports. During August 2014-September 2015, a total of 59 laboratories were approved to test for EBOV. This enhanced network consisted of state, large city, and metropolitan county public health laboratories (10). The ability to incorporate EBOV testing into existing processes and networks readily, as is the case with the LRN, is further evidence of past lessons learned and the value of strong national laboratory systems. Commercial availability of Food and Drug Administration (FDA)-authorized EBOV diagnostic tests is expanding these diagnostic services in facilities prepared to assess patients with suspected Ebola. Because of the consequences of a positive Ebola diagnosis and to ensure informed public health decision-making, the CDC reference laboratory must continue to confirm any presumptive positive Ebola diagnosis in these facilities.

\section{Diagnostic Testing Strategies and New Assay Developments}

Until this outbreak, few assays existed to detect, differentiate, and diagnose Ebola. The design and appropriate selection of diagnostic assays in Ebola evaluations depend on the patient's disease state. When the index of suspicion for Ebola is low but not negligible, ruling it out becomes a biosafety requirement because the presence of EBOV will lead to changes in the type of patient care needed, such as heightened precautions and limited laboratory testing to reduce exposure risks to medical and laboratory personnel.

During the acute viremic phase of illness, RT-PCR-based techniques are the most sensitive diagnostic method. They are frequently used with serology ( $\operatorname{IgM}$ and $\operatorname{IgG}$ ) to track virusnegative but antibody-positive survivors or for surveillance activities in geographic regions previously affected $(2,3)$. The timing of specimen collection in regard to symptom onset is key to evaluating any person suspected to have Ebola. During symptom onset, blood specimens are usually PCR positive; however, in a small number of patients, circulating virus titers might not reach detectable levels in peripheral blood for 72 hours, enabling potential false-negative results. For this reason, if symptoms have been present for $<3$ days, a second specimen might be required 72 hours after symptom onset to definitively rule out Ebola. Critically ill patients are highly viremic, and virus is readily detectable in oral swabs from deceased persons. In survivors, the humoral immune response begins to manifest toward the end of the second week of disease with transient $\operatorname{IgM}$ and rising $\operatorname{IgG}$ titers as circulating virus titers decrease (2).

The most frequently implemented diagnostic tests are based on quantitative RT-PCR (qRT-PCR) targeting conserved domains within genes for the viral polymerase (L) and structural elements (NP, VP40, and GP) of EBOV (species: Zaire ebolavirus). In October 2014, FDA issued emergency use authorizations (EUAs) for several EBOV RNA detection assays, including the DoD EZ1 Realtime RT-PCR, CDC's qRT-PCRs for the viral NP and VP40, and the bioMerieux BioFire Film array assay (BioFire Defense, LLC, Salt Lake City, Utah). In November 2014, Altona Diagnostics' RealStar Ebola Virus RT-PCR (Altona Diagnostics GmbH, Hamburg, Germany) followed, and in March 2015, Cepheid's Xpert Ebola Assay (Cepheid, Sunnyvale, California) was authorized for use on its GeneXpert platform. Details about these authorizations, the products, and their approved uses are available on the FDA website (13). Within the United States, these products are designated for use with patients demonstrating signs and symptoms of Ebola and require confirmatory testing. All are authorized for use in Clinical Laboratory Improvement Amendments (CLIA)-designated moderate-to-high-complexity 
laboratories with specific instrumentation. CDC's combined $\mathrm{NP}$ and VP40 assays typify the normal testing algorithm and incorporate an endogenous human housekeeping gene control for extraction and amplification controls. The presence of viral RNA is confirmed when both targets and the housekeeping gene are amplified and detected. This algorithm remains the confirmatory strategy for U.S. cases and for samples referred to CDC as a World Health Organization Collaborating Center for Viral Hemorrhagic Fevers.

These key molecular diagnostic assays are available for both domestic and international use by U.S. agencies, but they require staff qualified to perform moderate-to-high-complexity tests as well as modified biosafety protocols and complex workflows. The need for low-complexity, screening pointof-care assays to improve differential diagnosis and triage of suspected cases became evident early during the 2014-2016 Ebola epidemic. CDC worked closely with various partners and organizations to promote development of innovative assays able to support this requirement. A full array of diagnostic tests is under development in the public and private sectors.

In March 2015, FDA issued the first EUA for a lateral-flow antigen-capture assay to Corgenix, Inc. (Broomfield, Colorado) for the ReEBOV Antigen Capture Rapid Test. In July 2015, the OraQuick Ebola Rapid Antigen Test from OraSure Technologies, Inc. (Bethlehem, Pennsylvania) also was issued an EUA. These simple robust tests are based on the capture of circulating viral antigens by polyclonal or monoclonal antibodies bound to a filter strip and are driven by the wicking of the specimen (generally body fluids such as whole blood, plasma, or oral fluids) and reagents across the strip. The tests require no complex equipment; can be read in 30 minutes; and are individually packaged, stable, and disposable. Thus, these tests can be distributed widely as point-of-care assays in alternative testing sites (e.g., primary care and triage centers) that lack laboratory capacity. This technology also lends itself to multipathogen detection because several pathogens possibly can be captured on a single strip, which might provide differential diagnosis for confounding agents (e.g., malaria parasites, Marburg virus, and Lassa fever virus). Implementation of point-of-care testing brings challenges in training clinical staff in its use as well as in waste management, quality assurance, and development of alternative testing algorithms. The tests are approved for use on patients with symptoms consistent with Ebola and require further confirmation.

As further antiviral therapeutics or vaccines are deployed, particular attention must be given to selecting diagnostic assays and testing algorithms that can distinguish persons receiving these therapeutics from persons with natural virus infection. In West Africa, this difficulty is already recognized; however, with careful planning, appropriate reagents can be selected to avoid confusion and provide robust and reliable laboratory diagnostic services.

\section{Overcoming Challenges}

The 2014-2016 Ebola epidemic provided an opportunity to test years of preparedness that required extensive support from public and private health sectors, associations, and multiple federal entities, ranging from the point of service up to the national level. The epidemic highlighted numerous difficulties common during the initial phases of laboratory responses to high-consequence pathogens, such as viral hemorrhagic fevers. Among these were timely and appropriate transport of specimens, limited availability of experienced staff, integration of testing for public health and case management needs, assurance of continuity of laboratory services for routine patient care, inadequate standard operating procedures in institutions, and need for sustainable diagnostic testing and differential testing strategies. The need to detect and respond to a pathogen for which no commercially available assays were readily available further complicated these efforts.

Domestically, CDC was able to rely heavily on the administrative structures and processes in place with the LRN and state and local public health agencies while operational practices and safeguards were addressed in the health care setting. In the United States, rapid implementation of heightened biosafety practices and distribution of specialized testing capabilities and guidance were required to support the laboratory systems of both clinical and public health laboratories. Fortunately, the existing framework of a robust and adaptable laboratory system enabled effective deployment of assays and response. Challenges were mostly caused by biosafety concerns from the use of high-throughput instrumentation rather than by an inability of the laboratory system to absorb and adapt to change.

Internationally, the need for capacity building and lack of overall laboratory system capacity were pronounced and were addressed in parallel as the response was implemented. Most often the question was not what had to be done but rather how it should be accomplished on a scale appropriate to the needs of the response. There were additional challenges in coordinating a multinational laboratory response; supplementing the limited infrastructure resources to support laboratories; and communicating among all partners, including established ministry of health structures and nascent emergency operations centers in each country.

The need to expand timely testing to support informed patient management and public health decision-making was an ongoing concern. That resource-poor communities lacked 
supplies for safe collection and proper transport of specimens was recognized early by responders. This deficiency slowed specimen processing times because care was needed to avoid broken glass, needles, and other dangerous conditions. CDC and its partners supplied each country with hundreds of International Air Transport Association-compliant plastic specimen-transport containers and developed a pictogram illustrating proper packaging $(14,15)$. However, one underlying difficulty in fully addressing these needs was the lack of overall appreciation for the workflow processes common to all diagnostic testing: the preanalytical (e.g., sample collection, documentation, and transport), analytical, and postanalytical paths of work. A common consequence of this was use of the time from sample collection to aggregate data reporting as a measure of laboratory performance. This misunderstanding greatly hindered the process of identifying and rectifying preanalytical root causes, which are independent of the laboratory testing processes. Chief among these were inadequate documentation of time of symptom onset, patient clinical information, and time of sample transport to the laboratory. Failure to properly document symptom onset meant requests for retesting patients within the 72 -hour window were not followed up, and the inconsistent use of patient identifiers made linking repeat testing to patient monitoring and epidemiologic data difficult. In many instances, test results did not follow the patient, who might have moved into treatment away from local clinics or holding centers. This problem was of particular concern if patients were moved before results were reported through official channels and the laboratory had no direct contact with the care center or patient.

As the epidemic progressed, communications and transportation networks expanded, with varying degrees of success, to meet these needs. The most notable examples included rapid specimen transport by helicopters, where available, and national electronic reporting databases with ever-increasing fidelity of patient information. However, the overall number of tests done never approached the available testing capacity in the most-affected countries and was directly linked to low overall specimen collection activities and specimen transport.

\section{Conclusion}

West Africa's ability to develop long-term, sustainable laboratory capacity to recognize and respond to future threats to health security depends on improvements in the laboratory system. These improvements involve infrastructure reforms and organizational changes in laboratory networks, standardization of processes and procedures for rapid deployment of testing strategies, mechanisms to develop and adapt laboratory tools, and critical expansion of skilled workforce development as identified in the Global Health Security Agenda initiative (16).

Domestically, the U.S. public health laboratory infrastructure demonstrated its robust and flexible capacity to respond to a potential high-impact health emergency. However, further examination and refinement of biosafety and laboratory practices are needed to safely manage potentially hazardous patient specimens in today's complex laboratory environment. The integrated and close cooperation of the U.S. national, state, and local agencies and professional advisory groups during this response was a tremendous asset. Nevertheless, these interactions should be strengthened further to guard against the next high-consequence health threat.

Establishment of emergency operations centers in both domestic and international settings during the response substantially benefited laboratories. These coordinating centers provided feedback mechanisms to enable recognition of core issues and provide for process improvements that would be difficult to achieve in the rigid administrative structures under which laboratories often operate in resource-poor settings. The growing role of laboratory technical working groups within ministries of health and engagement with the African Society for Laboratory Medicine indicate the successful transitioning of these efforts into established structures. Nevertheless, these nascent efforts must be refined into more effective and efficient entities for coordinating and assisting emergency laboratory responses. Recognition by the affected countries that residual structures, such as mobile laboratories and diagnostic equipment donated in the response, must migrate into defined functional roles within the established national laboratory network is essential to capitalize on the advances implemented during this health emergency. Close cooperation between the many international agencies and partners involved with a focus on long-term development strategies will help prevent a repeat of the West Africa Ebola tragedy and provide for sustainable public health capacities throughout West Africa and beyond.

\section{Acknowledgement}

Ebola Laboratory Response Team: Amanda Balish, Dianna Blau, Michael Bowen, Aaron Brault, Shelley Brown, Deborah Cannon, Barry Fields, Aridth Gibbons, Ben Humrighouse, Kevin Karem, Gena Lawrence, William Lonegran, Laura McMullan, Toby Merlin, Stuart Nichol, Christopher Paddock, Gerald Pellegrini, Lance Presser, Pierre Rollin, John Saindon, Angela Sanchez, Victoria Jersey Scott, Josh Self, Martin Steinau, Robyn Stoddard, Jorn Winter, and Galina Zemtsova. 


\section{References}

1. World Health Organization. Ground zero in Guinea: the Ebola outbreak smoulders - undetected - for more than 3 months. http://www.who.int/ csr/disease/ebola/ebola-6-months/guinea/en

2. Peters CJ, LeDuc JW. An introduction to Ebola: the virus and the disease. J Infect Dis 1999;179(Suppl 1):ix-xvi. http://dx.doi.org/10.1086/514322

3. Towner JS, Rollin PE, Bausch DG, et al. Rapid diagnosis of Ebola hemorrhagic fever by reverse transcription-PCR in an outbreak setting and assessment of patient viral load as a predictor of outcome. J Virol 2004;78:4330-41. http://dx.doi.org/10.1128/JVI.78.8.4330-4341.2004

4. Towner JS, Khristova ML, Sealy TK, et al. Marburgvirus genomics and association with a large hemorrhagic fever outbreak in Angola. J Virol 2006;80:6497-516. http://dx.doi.org/10.1128/JVI.00069-06

5. Towner JS, Sealy TK, Ksiazek TG, Nichol ST. High-throughput molecular detection of hemorrhagic fever virus threats with applications for outbreak settings. J Infect Dis 2007;196(Suppl 2):S205-12. http://dx.doi.org/10.1086/520601

6. CDC. Guidance on personal protective equipment (PPE) to be used by healthcare workers during management of patients with confirmed Ebola or persons under investigation (PUIs) for Ebola who are clinically unstable or have bleeding, vomiting, or diarrhea in U.S. hospitals, including procedures for donning and doffing PPE. Atlanta, GA: US Department of Health and Human Services, CDC; 2016. http://www.cdc.gov/vhf/ ebola/healthcare-us/ppe/guidance.html

7. Flint M, Goodman CH, Bearden S, et al. Ebola virus diagnostics: the US Centers for Disease Control and Prevention laboratory in Sierra Leone, August 2014 to March 2015. J Infect Dis 2015;212(Suppl 2):S350-8. http://dx.doi.org/10.1093/infdis/jiv361

8. Karwowski MP, Meites E, Fullerton KE, et al. Centers for Disease Control and Prevention (CDC). Clinical inquiries regarding Ebola virus disease received by CDC-United States, July 9-November 15, 2014. MMWR Morb Mortal Wkly Rep 2014;63:1175-9.
9. Van Beneden CA, Pietz H, Kirkcaldy RD, et al. Early identification and prevention of the spread of Ebola-United States. In: CDC response to the 2014-2016 Ebola epidemic-West Africa and United States. MMWR Suppl 2016;65(No. Suppl 3).

10. CDC. Guidance for U.S. laboratories for managing and testing routine clinical specimens when there is a concern about Ebola virus disease Atlanta, GA: US Department of Health and Human Services, CDC; 2016. http://www.cdc.gov/vhf/ebola/healthcare-us/laboratories/safespecimen-management.html

11. CDC. Interim guidance for U.S. hospital preparedness for patients under investigation (PUIs) or with confirmed Ebola virus disease (EVD): a framework for a tiered approach. Atlanta, GA: US Department of Health and Human Services, CDC; 2016. http://www.cdc.gov/vhf/ebola/ healthcare-us/preparing/hospitals.html

12. CDC. The Laboratory Response Network partners in preparedness. Atlanta, GA: US Department of Health and Human Services, CDC; 2016. https://emergency.cdc.gov/lrn/

13. US Food and Drug Administration. Emergency use authorizations. http://www.fda.gov/EmergencyPreparedness/Counterterrorism/ MedicalCountermeasures/MCMLegalRegulatoryandPolicyFramework/ ucm 182568.htm\#ebola

14. CDC. Packaging and shipping clinical specimens diagram. Atlanta, GA: US Department of Health and Human Services, CDC; 2016. http://www. cdc.gov/vhf/ebola/healthcare-us/laboratories/shipping-specimens.html

15. World Health Organization. How to safely ship human blood samples from suspected Ebola cases within a country by road, rail and sea. http:// www.who.int/csr/resources/publications/ebola/blood-shipment/en

16. Global Health Securities Agenda. Action packages. http://www.ghsagenda.org 